\title{
Three-component olive oil-in-water high internal phase emulsions stabilized by palm surfactant and their moisturizing properties.
}

\begin{abstract}
In the present study, olive oil was used for the preparation of three-component high internal phase emulsions with oil volume fraction of more than 0.77 stabilized by palm-based polyoxyethylene lauryl ether for the first time. These emulsions were investigated on their morphology, structural properties, stability, and hydration efficacy. Droplet size distribution observed from the optical micrographs was in agreement with the light scattering results, which suggested that droplet size was influenced by oil phase and surfactant concentrations. Rheological results exhibiting flow curves cross-over implied structural build-up that gave rise to high stability which was supported by stable three-month storage at an elevated temperature. The hydration efficacy of the emulsion was examined in vivo using a corneometer.
\end{abstract}

Keyword: Corneometer; Light scattering; Oil-in-water high internal phase emulsion; Palmbased polyoxyethylene lauryl ether; Rheology. 